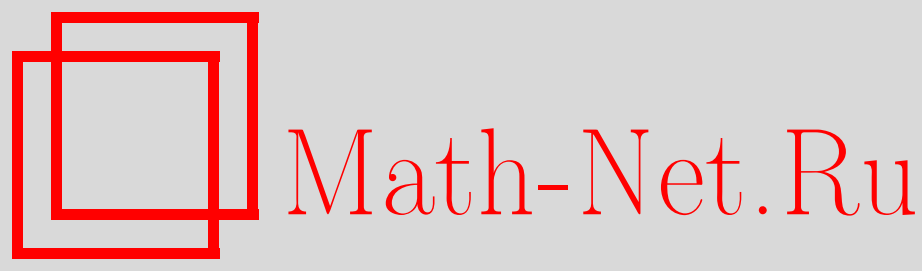

В. В. Поддубный, И. О. Глебов, С. М. Сударькова, Применимость приближения равновесности белкового окружения при описании сверхбыстрых биофизических процессов, ТМФ, 2015, том 183, номер 3, 498-512

DOI: https://doi.org/10.4213/tmf8840

Использование Общероссийского математического портала Math-Net.Ru подразумевает, что вы прочитали и согласны с пользовательским соглашением http://www.mathnet.ru/rus/agreement

Параметры загрузки:

IP: 54.80 .97 .219

26 апреля 2023 г., 13:52:01

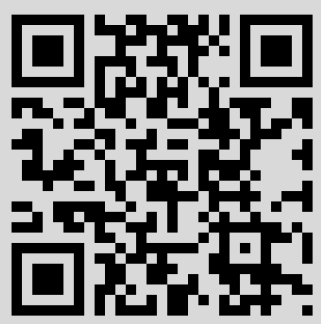




\title{
ПРИМЕНИМОСТЬ ПРИБЛИЖЕНИЯ РАВНОВЕСНОСТИ БЕЛКОВОГО ОКРУЖЕНИЯ ПРИ ОПИСАНИИ СВЕРХБЫСТРЫХ БИОФИЗИЧЕСКИХ ПРОЦЕССОВ
}

\begin{abstract}
Теоретическое описание сверхбыстрых процессов в биологических системах, в частности переноса электрона в фотосинтетических реакционных центрах, является важной задачей современной биологической физики. В силу того что эти процессы протекают в белковой среде, с которой возможен обмен энергией, для их описания необходимо применять методы квантовой теории открытых систем. Однако из-за высокой скорости и специфики белкового окружения основополагающие приближения теории могут не выполняться. Изучена применимость приближения неизменности состояния белкового окружения (термостата) для диссипативной динамики переноса заряда между молекулами-пигментами, входящими в состав реакционных центров. Для этого использованы модельные системы, параметры которых близки к реальным. Сделан вывод о том, что данное приближение справедливо для описания как монотонной, так и осциллирующей динамики реакционной подсистемы в крупных биологических молекулах. Рассмотрены различные механизмы термализации термостата и показано, что термализация термостата происходит не за счет внутримолекулярного перераспределения колебательной энергии в нем, а только за счет его взаимодействия с реакционной подсистемой.
\end{abstract}

Ключевые слова: квантовая теория открытых систем, редуцированная матрица плотности, термостат, фотосинтетический реакционный центр, перенос электрона.

DOI: $10.4213 / \operatorname{tmf} 8840$

\section{1. ВВЕДЕНИЕ}

Процессы переноса энергии и электрона играют одну из ключевых ролей в фотосинтетических системах - светособирающих антеннах и реакционных центрах. Зачастую такие процессы имеют очень высокую эффективность, которую обычно связывают со спецификой белковых систем [1] и с когерентным характером этих процессов [2]. Однако несмотря на огромное количество экспериментальных работ,

${ }^{*}$ Химический факультет, Московский государственный университет им. М. В. Ломоносова, Москва, Россия. E-mail: vvpoddubnyy@gmail.com, glebov_io@phys.chem.msu.ru, sudarkova.sveta@gmail.com 
посвященных изучению процессов переноса в фотосистемах, полученные результаты не могут достоверно прояснить механизмы этих процессов и выявить причину их высокой эффективности. В связи с этим актуальной является задача неэмпирического моделирования этих процессов.

Наибольший интерес для моделирования представляют первичные процессы, происходящие после поглощения фотосистемами энергии света. Они происходят с очень высокой скоростью и часто имеют когерентный характер; кроме того, о них имеется большое количество экспериментальных данных. В настоящей работе мы рассматриваем процесс первичного переноса электрона в реакционном центре фотосинтеза пурпурной бактерии Rh. sphaeroides. Этот процесс происходит практически необратимо и очень быстро - характерные времена переноса составляют несколько пикосекунд [3]. Фемтосекундная динамика образования состояния с разделенными зарядами характеризуется осцилляциями [4], природа которых до сих пор достоверно не выяснена. Существуют несколько объяснений этих осцилляций, из которых отметим модуляцию переноса электрона движением колебательного волнового пакета, образовавшегося при фотовозбуждении реакционного центра [4], и участие промежуточного электронного состояния в переносе электрона [5]. Эти объяснения основаны на квантовой теории открытых систем, где в качестве акцептора энергии (термостата) выступают колебательные степени свободы белкового окружения [6].

В рамках такого подхода фемтосекундные спектры поглощения и вынужденного излучения были смоделированы на основе предположения о том, что осцилляции вызваны движением колебательного волнового пакета [7]. Для описания динамики переноса электрона была использована теория Редфилда [8]-[11]. В этих работах согласие с экспериментальными данными было получено путем использования набора эмпирических параметров. Однако применимость теории Редфилда для описания таких процессов вызывает сомнения из-за высокой скорости процесса и из-за специфики белкового окружения.

Ранее мы показали, что марковское приближение может не выполняться в белковой среде [12]. Рассмотрев немарковскую динамику модельных систем, мы продемонстрировали, что осцилляции в фемтосекундных зависимостях могут объясняться немарковостью процесса - нерезонансным взаимодействием электронных уровней системы хромофоров (наблюдаемой подсистемы) с нормальными модами колебаний их белкового окружения (с термостатом).

Помимо марковского приближения вызывает сомнение применимость и второго основополагающего приближения теории Редфилда - приближения о равновесности термостата. В теории Редфилда термостат имеет непрерывный спектр частот. В белковой среде число атомов хотя и велико (десятки тысяч), но конечно, поэтому спектр колебательных энергий белка дискретен. Благодаря этому в ходе переноса электрона энергия может рассеиваться на небольшое число колебательных мод и тем самым изменять состояние термостата, делая его неравновесным.

Для выяснения корректности приближения равновесности термостата при описании диссипативной динамики в белковых средах мы рассмотрели модельные системы, параметры которых близки к простейшим реальным фотосинтетическим системам. Изучая динамику термостата в этих системах, мы исследовали возможные механизмы термализации термостата и показали, что она происходит не за счет внутримолекулярного перераспределения колебательной энергии в нем, а только за счет его взаимодействия с реакционной подсистемой. 


\section{2. МОДЕЛЬ ПЕРЕНОСА ЭЛЕКТРОНА В РЕАКЦИОННЫХ ЦЕНТРАХ ПУРПУРНЫХ БАКТЕРИЙ}

Для описания переноса электрона необходимо использовать теорию открытых квантовых систем. При таком описании в реакционном центре выделяют наблюдаемую подсистему, в качестве которой рассматриваются донор (специальная пара молекул бактериохлорофиллов) и акцептор (молекула мономерного бактериохлорофилла) электрона. Гамильтониан полной системы включает три слагаемых гамильтонианы наблюдаемой подсистемы и термостата, а также оператор, описывающий их энергию взаимодействия:

$$
\widehat{H}=\widehat{H}_{\mathrm{S}}+\widehat{H}_{\mathrm{B}}+\widehat{H}_{\mathrm{SB}} .
$$

В зависимости от интерпретации осцилляций в этой системе выбирают набор уровней (электрон-колебательных [7] или чисто электронных [12]), переходы между которыми и определяют скорость переноса электрона. Вторая часть реакционного центра - белковое окружение реакционной подсистемы хромофоров - должна выступать в роли термостата, который должен поглощать энергию, выделяющуюся при переносе электрона. Эта энергия не очень велика (примерно $550 \mathrm{~cm}^{-1}$ ) и недостаточна для возбуждения электронных уровней белкового окружения, но примерно равна энергиям переходов между колебательными уровнями. Поэтому при описании динамики в термостате рассматриваются исключительно колебательные уровни белкового окружения [6]:

$$
\widehat{H}_{\mathrm{B}}=\sum_{k} \hbar \omega_{k}\left(\hat{a}_{k}^{+} \hat{a}_{k}+\frac{1}{2}\right)
$$

где $\omega_{k}$ - частота $k$-й моды, а $\hat{a}_{k}^{+}$и $\hat{a}_{k}$ - операторы рождения и уничтожения, действующие на состояния $k$-й моды.

Взаимодействие "система-термостат" описывается либо как фонон-фононное [7], либо как электрон-фононное [12]. В любом случае оператор взаимодействия в приближении слабости взаимодействия представляется в виде, линейном по смещению атомов белкового окружения:

$$
\widehat{H}_{\mathrm{SB}}=\sum_{k} \hat{F}_{k} \hat{x}_{k}
$$

где $\hat{F}_{k}$ - оператор, действующий на наблюдаемую подсистему, а $\hat{x}_{k}$ - оператор координаты $k$-й нормальной моды колебания белкового окружения, который может быть представлен в виде суммы операторов рождения и уничтожения:

$$
\hat{x}_{k}=\sqrt{\frac{\hbar}{2 \omega_{k} m_{k}}}\left(\hat{a}_{k}^{+}+\hat{a}_{k}\right),
$$

где $m_{k}$ - приведенная масса $k$-й моды. С учетом такой замены можно переопределить оператор взаимодействия:

$$
\widehat{H}_{\mathrm{SB}}=\sum_{k} \hat{g}_{k}\left(\hat{a}_{k}^{+}+\hat{a}_{k}\right),
$$


где оператор $\hat{g}_{k}$ определяет интенсивность взаимодействия наблюдаемой подсистемы с $k$-й модой термостата. Матричные элементы этого оператора в базисе собственных состояний наблюдаемой подсистемы $|I\rangle,|J\rangle$ можно представить в виде спектральных функций [9]:

$$
J_{I, J}(\omega)=\sum_{k}\left\langle I\left|\hat{g}_{k}\right| J\right\rangle^{2} \delta\left(\omega-\omega_{k}\right)
$$

Мы придерживаемся точки зрения, согласно которой перенос электрона является чисто электронным процессом в рамках наблюдаемой подсистемы, т. е. при переносе энергии колебания молекул хромофоров не влияют на динамику переноса, а равновесное положение этих атомов в возбужденных состояниях очень слабо отличается от их равновесного положения в основном электронном состоянии. В простейшем случае в наблюдаемой подсистеме рассматривается переход между двумя ее состояниями - возбужденным состоянием и состоянием с перенесенным электроном (основное состояние в рассматриваемом процессе участия не принимает). Тогда гамильтониан наблюдаемой подсистемы представляется в виде

$$
\widehat{H}_{\mathrm{S}}=|0\rangle E_{0}\langle 0|+| 1\rangle E_{1}\langle 1|,
$$

где $|0\rangle$ и $|1\rangle$ - состояние с перенесенным электроном и возбужденное состояние системы соответственно, а $E_{0}$ и $E_{1}-$ их энергии (состояния пронумерованы в порядке увеличения энергии). Мы предполагаем, что диагональные элементы операторов $\hat{g}_{k}$ равны нулю, следовательно, динамика переноса определяется их недиагональными элементами.

Неэмпирический расчет матричных элементов операторов $\hat{g}_{k}$ крайне сложен, поскольку для этого требуется знание электронных волновых функций наблюдаемой подсистемы и нормальных мод колебания белкового окружения [13]. Поэтому при моделировании переноса электрона спектральную функцию описывают в виде гладкой функции, в частности омической с экспоненциальным срезом [6], [9]:

$$
J(\omega)=\eta \omega e^{-\omega / \omega_{c}},
$$

где параметр $\eta$ - амплитуда взаимодействия "система-термостат", а частота $\omega_{\mathrm{c}}$ определяет положение максимума функции.

Однако из выражений (3)-(6) следует, что спектральная функция должна иметь асимптотику $1 / \omega$, так как операторы $\hat{F}_{k}$ не зависят от частоты $k$-го колебания. Потому вместо (8) мы использовали функцию вида [14]

$$
J(\omega)=2 A \frac{\omega_{\mathrm{c}} \omega}{\omega^{2}+\omega_{\mathrm{c}}^{2}},
$$

где $A$ - максимальное значение спектральной функции. Использование такой спектральной функции не приводит к осцилляциям заселенности состояния наблюдаемой подсистемы с перенесенным электроном, но позволяет описывать ее рост, т. е. диссипацию энергии наблюдаемой подсистемы [12]. Для того чтобы в динамике наблюдались осцилляции, необходимо, чтобы в спектральной функции существовали узкие пики с максимумами на частотах, не равных частоте перехода в наблюдаемой 
подсистеме [12]. Для воспроизведения такого эффекта в приближенную спектральную функцию (9) можно добавить гауссову функцию:

$$
J(\omega)=2 A \frac{\omega_{\mathrm{c}} \omega}{\omega^{2}+\omega_{\mathrm{c}}^{2}}+\eta e^{-(\omega-\widetilde{\omega})^{2} / 2 \sigma^{2}}
$$

где $\eta$ определяет амплитуду осцилляций, $\sigma$ определяет ширину пика, а разность $\widetilde{\omega}$ и частоты перехода в наблюдаемой подсистеме $\left(\omega_{\mathrm{S}}\right)$ определяет частоту этих осцилляций.

При рассмотрении применимости приближения равновесности термостата мы будем использовать спектральные функции (9) и (10) и рассматривать процесс как чисто электронный, однако полученные результаты могут быть распространены и на случай колебательного движения хромофоров в процессе переноса электрона.

\section{3. ЗАВИСИМОСТЬ АМПЛИТУДЫ ВЗАИМОДЕЙСТВИЯ МОД ТЕРМОСТАТА С НАБЛЮДАЕМОЙ ПОДСИСТЕМОЙ ОТ ЧАСТОТЫ}

Наблюдаемая подсистема при переходе из возбужденного состояния в состояние с перенесенным электроном рассеивает энергию по некоторому количеству мод термостата. Логично предположить, что наиболее сильно при этом наблюдаемая подсистема взаимодействует с модами, частоты которых близки к резонансной частоте, соответствующей разности энергий между рассматриваемыми состояниями наблюдаемой подсистемы. Немарковость процесса [12] означает, что необходимо также учитывать нерезонансные взаимодействия с колебаниями, для которых частоты $\omega$ не равны $\omega_{\mathrm{S}}$. Поэтому возникает вопрос о зависимости амплитуды взаимодействия системы с колебательными модами термостата от частоты последних.

Рассмотрим простейшую систему, в которой донор и акцептор имеют по два состояния (основное и возбужденное) и взаимодействуют между собой. Гамильтониан такой модельной системы может быть представлен в виде суммы гамильтонианов отдельных систем и оператора, описывающего их взаимодействие:

$$
\begin{aligned}
\widehat{H}= & |0\rangle_{1} 0\left\langle\left. 0\right|_{1}+\mid 1\right\rangle_{1} \hbar \omega_{1}\left\langle\left. 1\right|_{1}+\mid 0\right\rangle_{2} 0\left\langle\left. 0\right|_{2}+\mid 1\right\rangle_{2} \hbar \omega_{2}\left\langle\left. 1\right|_{2}+\right. \\
& +\hbar g\left(| 0 \rangle _ { 1 } \langle 1 | _ { 1 } + | 1 \rangle _ { 1 } \langle 0 | _ { 1 } ) \left(|0\rangle_{2}\left\langle\left. 1\right|_{2}+\mid 1\right\rangle_{2}\left\langle\left. 0\right|_{2}\right),\right.\right.
\end{aligned}
$$

где первые четыре слагаемых описывают гамильтонианы изолированных донора и акцептора ( $\omega_{1}$ и $\omega_{2}-$ их частоты, а энергии их нулевых уровней приняты за ноль), а последнее слагаемое описывает их взаимодействие, которое характеризуется параметром $g$.

Предположим, что в начальный момент система находится в состоянии $\psi(0)=$ $|1\rangle_{1}|0\rangle_{2}$. В рассматриваемой системе нет никаких путей диссипации, а начальное состояние является чистым, следовательно, в любой момент времени состояние можно описать с помощью волновой функции. Оператор взаимодействия построен так, что переводит начальное состояние $\psi(0)=|1\rangle_{1}|0\rangle_{2}$ в состояние $|0\rangle_{1}|1\rangle_{2}$ и обратно, но он не переводит ее в состояния, в которых обе подсистемы одновременно находятся в возбужденном или в основном состояниях $\left(|1\rangle_{1}|1\rangle_{2}\right.$ и $|0\rangle_{1}|0\rangle_{2}$ соответственно). Поэтому мы можем ограничиться рассмотрением только двух состояний, участвующих 
в процессе:

$$
|\mathrm{I}\rangle=|1\rangle_{1}|0\rangle_{2}, \quad|\mathrm{II}\rangle \quad=|0\rangle_{1}|1\rangle_{2} .
$$

В базисе этих состояний гамильтониан всей системы принимает вид

$$
\widehat{H}_{\text {full }}=|\mathrm{I}\rangle \hbar \omega_{1}\langle\mathrm{I}|+| \mathrm{II}\rangle \hbar \omega_{2}\langle\mathrm{II}|+\hbar g(|\mathrm{I}\rangle\langle\mathrm{II}|+| \mathrm{II}\rangle\langle\mathrm{I}|) .
$$

Динамика этой системы зависит не от абсолютных значений каждой из частот, а от их разности $\left(\Delta \omega=\omega_{2}-\omega_{1}\right)$, поэтому можно принять $\omega_{1}=0$.

В любой момент времени состояние системы описывается волновой функцией

$$
\psi(t)=c_{\mathrm{I}}(t)|\mathrm{I}\rangle+c_{\mathrm{II}}(t)|\mathrm{II}\rangle
$$

Подставив эту волновую функцию во временно́е уравнение Шредингера, получаем систему уравнений с начальными условиями:

$$
\begin{aligned}
i \dot{c}_{\mathrm{I}}(t) & =g c_{\mathrm{II}}(t), \\
i \dot{c}_{\mathrm{II}}(t) & =\Delta \omega c_{\mathrm{II}}(t)+g c_{\mathrm{I}}(t), \\
c_{\mathrm{I}}(0) & =1, \quad c_{\mathrm{II}}(0)=0 .
\end{aligned}
$$

Продифференцировав уравнение на $\dot{c}_{\mathrm{II}}(t)$ и подставив в него $\dot{c}_{\mathrm{I}}(t)$, получим однородное дифференциальное уравнение второго порядка

$$
i \ddot{c}_{\mathrm{II}}(t)=\Delta \omega \dot{c}_{\mathrm{II}}(t)-i g^{2} c_{\mathrm{II}}(t) .
$$

Решение этого уравнения имеет общий вид

$$
c_{\mathrm{II}}(t)=A \exp \left[\frac{i}{2}\left(\Delta \omega+\sqrt{\Delta \omega^{2}+4 g^{2}}\right) t\right]+B \exp \left[\frac{i}{2}\left(\Delta \omega-\sqrt{\Delta \omega^{2}+4 g^{2}}\right) t\right] .
$$

Из начальных условий следует

$$
c_{\mathrm{II}}(t)=-\frac{2 i g e^{i \Delta \omega t / 2}}{\sqrt{\Delta \omega^{2}+4 g^{2}}} \sin \left(\sqrt{\Delta \omega^{2}+4 g^{2}} t\right) .
$$

Из полученного выражения следует, что заселенность возбужденного состояния акцептора энергии зависит от времени следующим образом:

$$
P_{\mathrm{II}}(t)=\frac{4 g^{2}}{\Delta \omega^{2}+4 g^{2}} \sin ^{2}\left(\sqrt{\Delta \omega^{2}+4 g^{2}} t\right) .
$$

В итоге максимальное значение заселенности возбужденного состояния системы II (акцептора энергии) зависит от разности частот следующим образом:

$$
\max _{t} P_{\mathrm{II}}=\frac{4 g^{2}}{4 g^{2}+\left(\omega_{1}-\omega_{2}\right)^{2}}
$$

Полученная зависимость показывает, что ширина области частот, с которыми наиболее сильно взаимодействует наблюдаемая подсистема, определяется параметpoм $g$, т. е. фактически амплитудой взаимодействия. Полуширина этой области равна $|2 g|$. 
Рассмотрим наблюдаемую подсистему, взаимодействующую с термостатом, имеющим равномерное распределение частот в спектре. Предположим, мы увеличиваем плотность распределения в $N$ раз, не меняя аппроксимацию спектральной функции (например (8) или (9)). Тогда число слагаемых в определении спектральной функции (6) увеличивается в $N$ раз, следовательно, каждый матричный элемент уменьшается в $\sqrt{N}$ раз. Соответственно в $\sqrt{N}$ раз уменьшится и полуширина области частот, с которыми наиболее сильно взаимодействует подсистема. Однако из-за увеличения плотности распределения в эту область попадет в $\sqrt{N}$ раз больше колебательных мод.

При непрерывном распределении $N \rightarrow \infty$, а значит, в той или иной степени будет взаимодействовать с подсистемой очень большое число мод, поэтому состояния каждой из них и термостата в целом изменятся бесконечно слабо.

Таким образом, при непрерывном распределении частот предположение о неизменности термостата справедливо. Проверим это предположение для большого, но конечного числа частот.

\section{4. ДИНАМИКА ЗАСЕЛЕННОСТИ ВОЗБУЖДЕННЫХ СОСТОЯНИЙ МОД ТЕРМОСТАТА}

Для проверки этого предположения необходимо знать параметры спектральной функции, частоту перехода в наблюдаемой подсистеме и распределение частот. В простейшей модельной системе частота перехода системы соответствует разности энергий между возбужденным состоянием и состоянием с перенесенным электроном в реакционном центре фотосинтеза бактерии Rh. sphaeroides, которая равна $\sim 550 \mathrm{~cm}^{-1}$. Параметры спектральной функции можно подобрать таким образом, чтобы скорость рассматриваемого процесса примерно совпадала с экспериментально определенной. В данной работе мы используем равномерное распределение частот термостата, при котором число мод на $1 \mathrm{~cm}^{-1}$ одно и то же во всем спектральном диапазоне. Частоты мод термостата при этом определяются уравнением $\omega_{k}=(k+1) \Delta \omega$.

Согласно результатам расчета первых четырех тысяч нормальных мод колебаний белка в реакционном центре бактерии Rh. sphaeroides [13] такое приближение справедливо, а плотность распределения примерно равна 30 модам на $1 \mathrm{~cm}^{-1}$ для низкочастотной области частот от 0 до $130 \mathrm{~cm}^{-1}$. Это значение выше среднего значения 11 мод на $1 \mathrm{~cm}^{-1}$ по всему спектральному диапазону (количество колебательных степеней свободы реакционного центра равно примерно 42000, а спектр частот ограничен сверху значением $3800 \mathrm{~cm}^{-1}$ ). Такое расхождение объясняется тем, что в белковых молекулах в области частот 1900-2800 $\mathrm{cm}^{-1}$ число колебаний мало.

Основываясь на этих данных, будем предполагать, что плотность распределения колебательных частот белкового окружения вблизи значения $550 \mathrm{~cm}^{-1}$ не меньше 10 мод на $1 \mathrm{~cm}^{-1}$, и соответственно $\Delta \omega<0.1 \mathrm{~cm}^{-1}$. В расчетах мы использовали значения $\Delta \omega=1.17,2.34$ и $4.70 \mathrm{~cm}^{-1}$, которые более чем на порядок превышают оценочное значение $\Delta \omega$ для реальных систем. Выбор завышенных значений $\Delta \omega$ позволяет нам судить о применимости рассматриваемого приближения, поскольку в случае выполнения этого приближения для моделирования динамики таких модельных систем оно гарантированно будет применимо и при описании динамики в реальных системах. 
Для проверки применимости рассматриваемого приближения необходимо иметь точное решение уравнения фон Неймана для полной матрицы плотности всей системы, включая термостат:

$$
\frac{\partial}{\partial t} \hat{\rho}(t)=\frac{1}{i \hbar}[\widehat{H}, \hat{\rho}(t)]
$$

Даже при небольшом числе мод термостата решение такой задачи технически сложно, поэтому мы ограничимся рассмотрением только тех состояний, в которых лишь одна из мод термостата возбуждена, а все остальные находятся в основном состоянии. При этом мы учтем только основные и однократно возбужденные состояния для каждой моды.

4.1. Монотонная динамика. Рассмотрим сначала более простой случай, в котором мы используем спектральную функцию вида (9) с параметрами $A=0.1$ мэВ и $\omega_{\mathrm{c}}=712 \mathrm{~cm}^{-1}$. В наблюдаемой подсистеме рассматриваются только два состояния, а ее гамильтониан описывается формулой (7). Энергетическая разность между уровнями наблюдаемой подсистемы равна $588 \mathrm{~cm}^{-1}$. Мы рассмотрели модельные системы с различной плотностью распределения мод термостата, частоты которых определялись согласно уравнению $\omega_{k}=(k+1) \Delta \omega$. Параметры $\Delta \omega$ для различных систем были равны 1.17, 2.34 и $4.70 \mathrm{~cm}^{-1}$. Динамика системы была получена с помощью численного решения уравнения Лиувилля-фон Неймана (20) для полной матрицы плотности в базисе состояний, в которых лишь одна мода термостата или наблюдаемая система находится в возбужденном состоянии $(|S\rangle$ - возбужденное состояние наблюдаемой подсистемы, $|k\rangle$ - возбужденное состояние $k$-й моды термостата). В таком базисе заселенность возбужденного состояния наблюдаемой моды равна матричному элементу матрицы плотности $P_{\mathrm{S}}(t)=\langle S|\hat{\rho}(t)| S\rangle$, а заселенность возбужденного состояния $k$-й моды термостата равна $P_{k}(t)=\langle k|\hat{\rho}(t)| k\rangle$.

Расчеты показывают, что динамика заселенности возбужденного состояния наблюдаемой подсистемы очень слабо изменяется при увеличении плотности распределения мод термостата (рис. 1), несмотря на то что заселенность возбужденных состояний некоторых мод превышает $10 \%$ при малой плотности распределения. Поэтому при расчете динамики наблюдаемой подсистемы можно использовать приближение о неизменности состояния термостата.

Как видно на рис. 1, заселенность возбужденного состояния спадает монотонно и имеется индукционный период. Помимо индукционного периода в динамике не наблюдается никаких особенностей.

На рис. 2 показаны максимальные значения заселенности возбужденных состояний мод термостата для систем, в которых плотность распределения соответствует значениям $\Delta \omega=1.17,2.34$ и $4.70 \mathrm{~cm}^{-1}$. Данная гистограмма показывает, что при увеличении плотности распределения количество мод термостата, взаимодействующих с наблюдаемой подсистемой, увеличивается, что согласуется с результатом, полученным в конце раздела 3 . В результате с учетом того, что в расчетах использовалась плотность распределения, которая на порядок меньше, чем оценочное значение плотности реальных систем $\left(\Delta \omega_{\text {real }}<0.1 \mathrm{~cm}^{-1}\right)$, а значение заселенности возбужденного состояния отдельных мод термостата не превышает $3 \%$ в случае $\Delta \omega=1.17 \mathrm{~cm}^{-1}$, мы можем сделать вывод, что использование приближения неизменности состояния мод термостата применимо для биологических систем, 


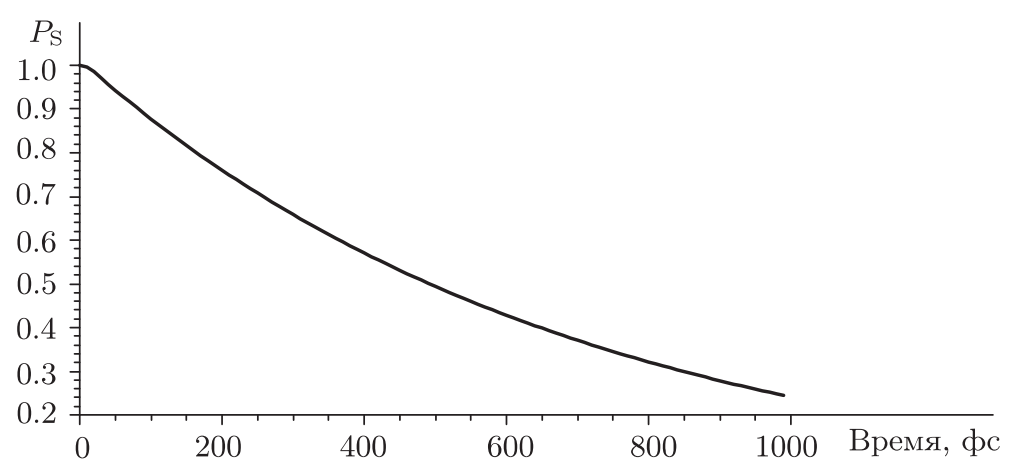

Рис. 1. Динамика заселенности возбужденного состояния наблюдаемой подсистемы $P_{\mathrm{S}}$ при значениях $\Delta \omega=1.17,2.34$ и $4.70 \mathrm{~cm}^{-1}$. Полученные кривые практически совпадают.

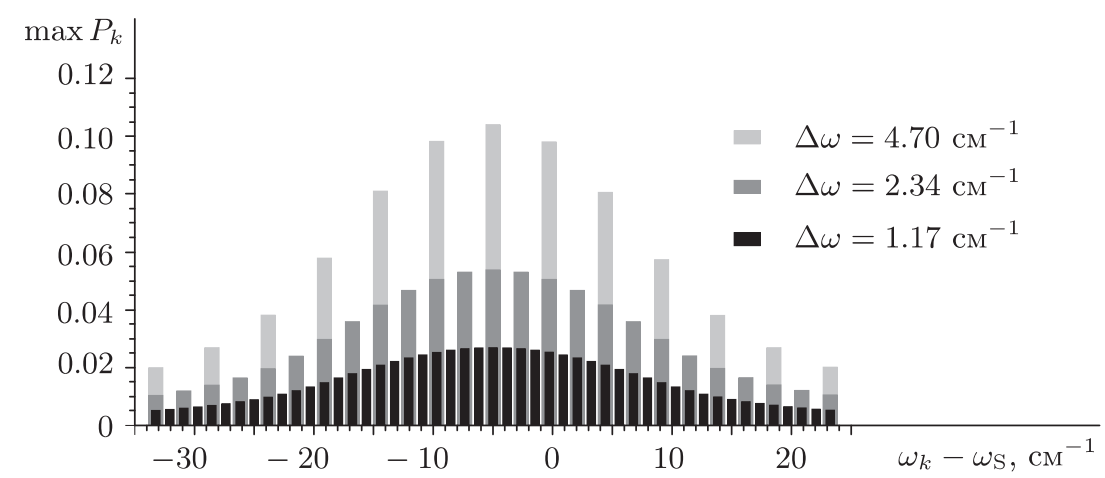

Рис. 2. Максимальные значения заселенности возбужденных состояний мод термостата $P_{k}$. Светло-серым цветом показаны данные для $\Delta \omega=4.70 \mathrm{~cm}^{-1}$, темно-серым цветом - данные для $\Delta \omega=2.34 \mathrm{~cm}^{-1}$, черным цветом - данные для $\Delta \omega=1.17 \mathrm{~cm}^{-1}$.

спектральная функция взаимодействия "система-термостат" которых схожа со спектральной функцией вида (9).

4.2. Динамика с осцилляциями. Однако спектральная функция взаимодействия "система-термостат" в реальных системах может быть более сложной, чем функция (9) [13]. В ней могут присутствовать пики, соответствующие взаимодействию колебаний ближайшего белкового окружения с наблюдаемой подсистемой хромофоров. Такие пики спектральной функции могут иметь максимумы на частотах, не совпадающих с частотой перехода в наблюдаемой подсистеме, но из-за высокого значения параметров $\left\langle 1\left|\hat{g}_{k}\right| 0\right\rangle^{2}$ эти моды могут сильно взаимодействовать с наблюдаемой подсистемой и, следовательно, заметно изменять свое состояние с течением времени.

Для учета этого мы рассчитали динамику системы, аналогичной рассмотренной в п. 4.1, но со спектральной функцией взаимодействия "наблюдаемая подсистема- 


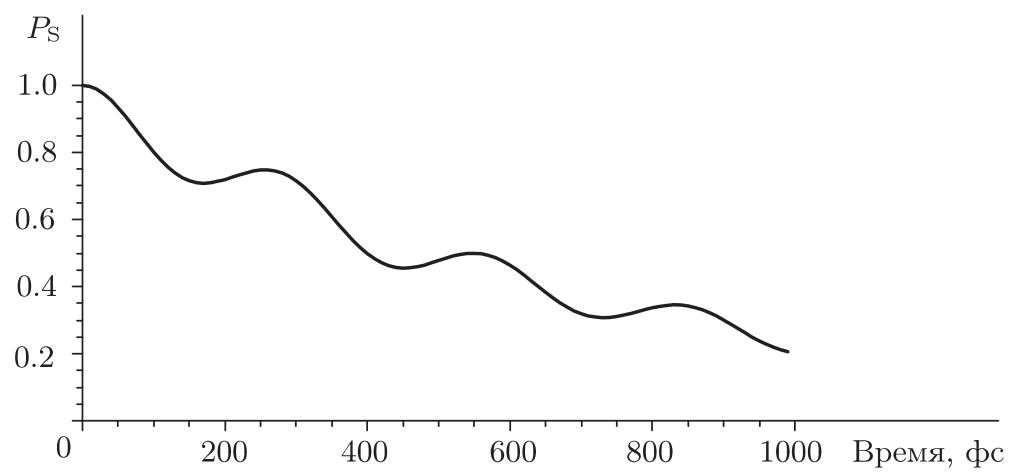

Рис. 3. Динамика заселенности возбужденного состояния наблюдаемой подсистемы в системе со спектральной функцией взаимодействия "система-термостат" вида (10). Параметры приведены в тексте.

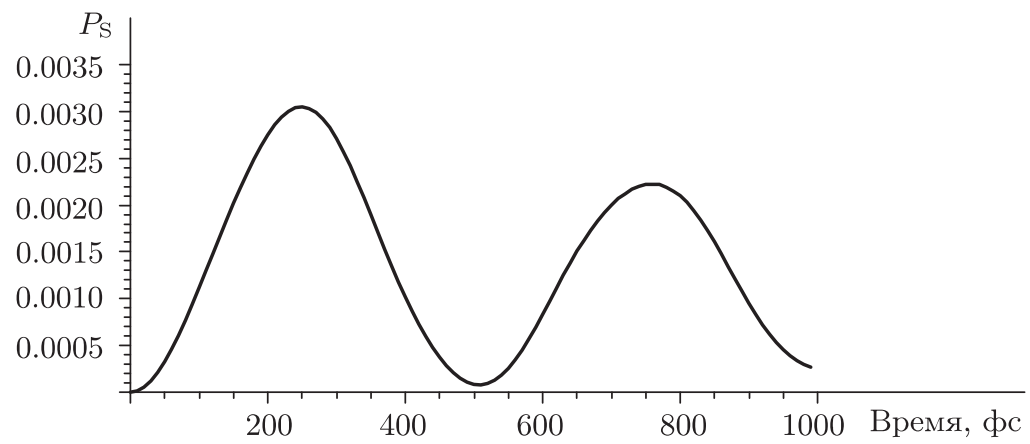

Рис. 4. Динамика заселенности возбужденного состояния моды, которая наиболее сильно взаимодействует с наблюдаемой подсистемой и обусловливает пик спектральной функции.

термостат" вида (10). На рис. 3 изображена зависимость заселенности возбужденного состояния от времени для такой системы с параметрами спектральной функции $A=0.1$ мэВ, $\omega_{\mathrm{c}}=712 \mathrm{~cm}^{-1}, \eta=5$ мэВ, $\widetilde{\omega}=694 \mathrm{~cm}^{-1}, \sigma=4 \mathrm{~cm}^{-1}$ и $\Delta \omega=1.17 \mathrm{~cm}^{-1}$.

Осцилляции заселенности возбужденного состояния наблюдаются не только в динамике наблюдаемой подсистемы, но и в заселенности возбужденного состояния мод, обусловливающих пик спектральной функции (рис. 4). На рассчитанной зависимости заселенности возбужденного состояния наблюдаемой подсистемы (рис. 3) также не появляется дополнительных эффектов по сравнению с результатами расчета динамики с использованием приближения неизменности состояния термостата.

На рис. 5 показаны максимальные значения заселенности возбужденных состояний мод термостата, обусловливающих пик спектральной функции. Из этой зависимости видно, что максимальное значение заселенности возбужденного состояния мод термостата не превышает $2.5 \%$, в то время как плотность распределения мод 


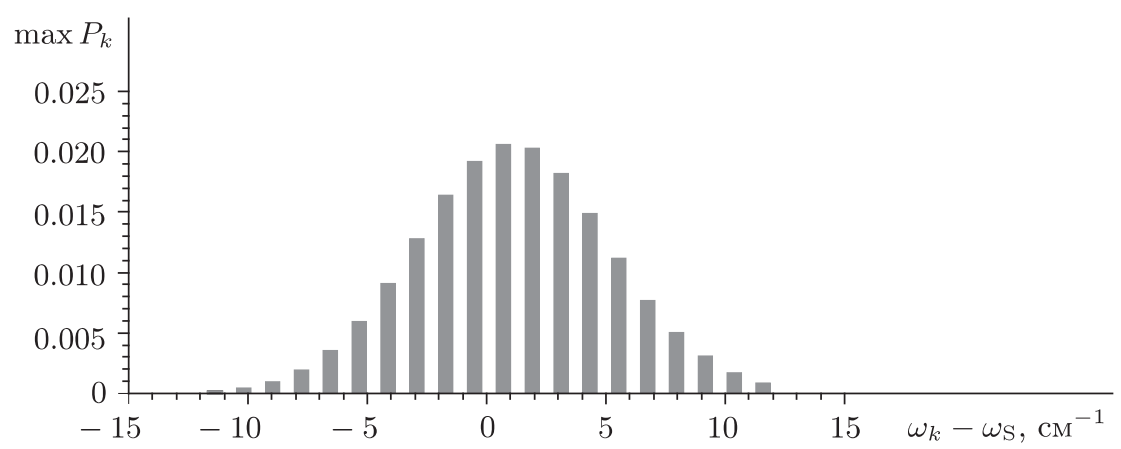

Рис. 5. Максимальные значения заселенности возбужденных состояний мод термостата, соответствующих пику спектральной функции.

термостата на порядок отличается от оценочного значения плотности реальных биологических систем.

На основе этого результата и результатов, полученных в п. 4.1, мы делаем вывод о том, что использование приближения неизменности состояния мод термостата справедливо для описания диссипативной динамики крупных биофизических систем при различных спектральных функциях и разных типах динамики наблюдаемой подсистемы. Естественно, состояние мод термостата в ходе динамики изменяется, но это не приводит к значимому изменению динамики наблюдаемой подсистемы.

\section{5. ТЕРМАЛИЗАЦИЯ ТЕРМОСТАТА}

Хотя состояние термостата не оказывает заметного влияния на динамику наблюдаемой подсистемы, представляет интерес вопрос о механизме термализации мод белка. При построении модели полной системы термостат представляется как набор гармонических осцилляторов, но в реальных системах гармоническое приближение может не выполняться. Поэтому в качестве первой гипотезы мы рассмотрели термализацию мод через их прямое взаимодействие между собой.

5.1. Термализация мод посредством прямого взаимодействия друг с другом. Для того чтобы проверить гипотезу о термализации термостата за счет прямого взаимодействия мод, мы рассмотрели динамику системы, аналогичной рассмотренной в п. 4.1, в гамильтониан термостата которой были добавлены элементы, описывающие взаимодействие мод:

$$
\widehat{H}_{\mathrm{B}_{\text {new }}}=\widehat{H}_{\mathrm{B}}+\sum_{k, l}|k\rangle V\langle l|,
$$

где $|k\rangle$ и $|l\rangle$ - состояния термостата, отвечающие возбуждению $k$-й и $l$-й мод, а параметр $V$ описывает амплитуду их взаимодействия.

Мы рассмотрели динамику системы с плотностью распределения частот, соответствующей значению $\Delta \omega=1.17 \mathrm{~cm}^{-1}$. Параметр $V$ был равен 0.1 мэВ и сравним со средним значением коэффициентов $\left\langle 0\left|\hat{g}_{k}\right| 1\right\rangle$. Зависимость заселенности возбужденного состояния наблюдаемой подсистемы представлена на рис. 6 сплошной линией. 


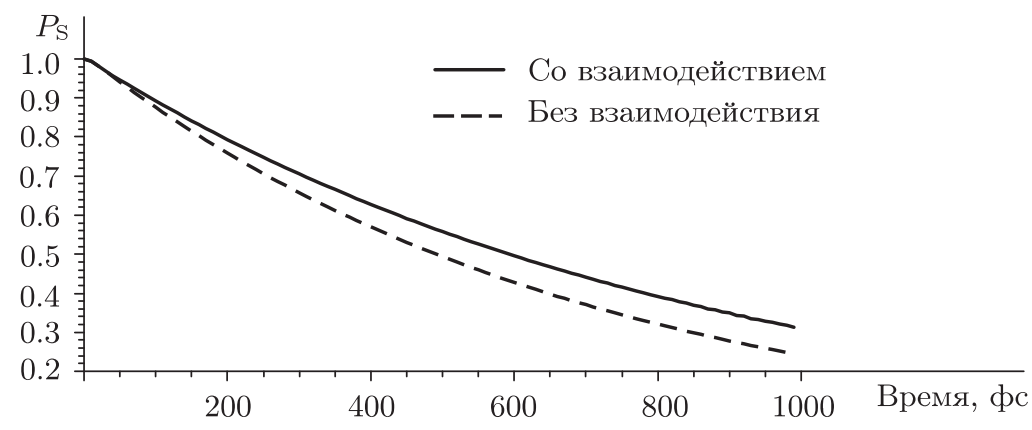

Рис. 6. Динамика заселенности возбужденного состояния наблюдаемой подсистемы. Штриховой линией показаны результаты, полученные с нулевым параметром $V$, сплошной линией показаны результаты, полученные с использованием параметра $V=0.1$ мэВ.

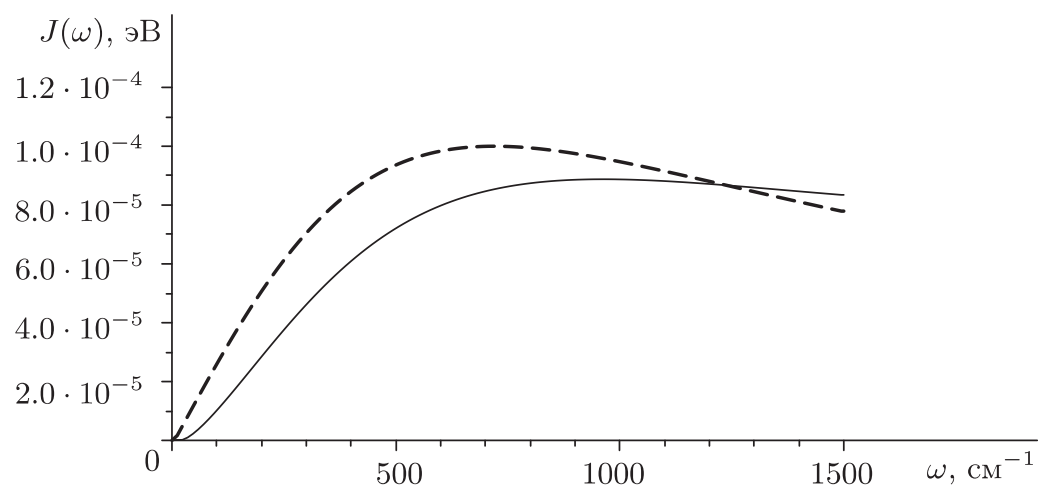

Рис. 7. Спектральные функции взаимодействия "система-термостат". Штриховой линией показана начальная спектральная функция, сплошной линией показана спектральная функция, переопределенная с учетом взаимодействия мод термостата.

Из сравнения кривых видно, что внесение дополнительных элементов в гамильтониан полной системы приводит к значительному изменению динамики наблюдаемой подсистемы. Такой результат вызван тем, что при изменении гамильтониана термостата меняются его собственные функции и, следовательно, спектральная функция.

Взаимодействие мод термостата в такой модели меняет собственные состояния и энергии термостата, которые могут быть получены путем нахождения собственных функций гамильтониана $\widehat{H}_{\mathrm{B}_{\text {new }}}$. В таком случае при переходе в новый базис состояний термостата необходимо переопределить спектральную функцию согласно (6). На рис. 7 представлено сравнение начальной спектральной функции с функцией, переопределенной с учетом взаимодействия мод термостата. 


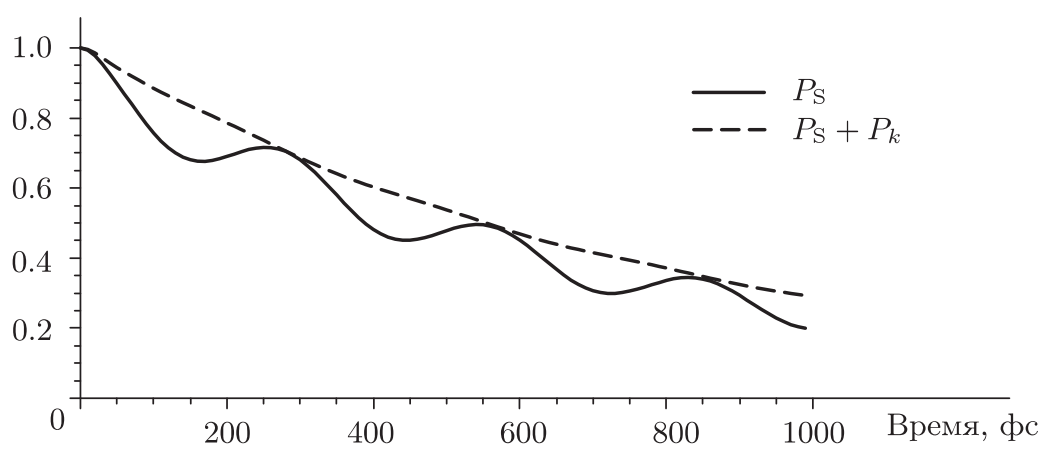

Рис. 8. Динамика заселенности возбужденного состояния наблюдаемой подсистемы (сплошная линия). Штриховой линией показана временна́я зависимость суммы заселенностей возбужденных состояний наблюдаемой подсистемы и выделенной моды термостата.

Стоит отметить, что при взаимодействии мод термостата изменяется не только итоговая спектральная функция взаимодействия "система-термостат", но и собственные энергии колебательных уровней. Этот эффект может сильно повлиять на динамику в случае, когда имеются одна или несколько выделенных мод термостата, которые сильно взаимодействуют с наблюдаемой подсистемой и обусловливают осцилляции в динамике заселенностей.

Таким образом, полученный результат показывает, что при описании диссипативной динамики биологических систем необходимо корректно выбирать гамильтониан термостата, учитывая при его построении ангармоническое взаимодействие колебаний белкового окружения.

\section{2. Термализация мод посредством их взаимодействия с наблюдаемой}

подсистемой. Учитывая полученный результат, можно предположить, что при корректном определении термостата единственным путем его термализации остается взаимодействие мод друг с другом через наблюдаемую подсистему. Для проверки этой гипотезы мы рассмотрели систему из п. 4.1, в термостат которой мы добавили одну сильно взаимодействующую с наблюдаемой подсистемой моду. В реальной системе такой моде может соответствовать колебание ближайших к реакционной подсистеме аминокислотных остатков. Частота этой моды отличается от частоты перехода в системе на $106 \mathrm{~cm}^{-1}$, параметр $\langle 0|\hat{g}| 1\rangle$, соответствующий этой моде, равен 3 мэВ. В динамике заселенности возбужденного состояния наблюдаемой подсистемы в этом случае наблюдаются осцилляции, амплитуда которых совпадает с амплитудой осцилляций заселенности возбужденного состояния выделенной моды термостата. При этом фазы этих осцилляций отличаются так, что сумма заселенностей спадает монотонно (рис. 8).

Амплитуда этих осцилляций в начальный промежуток времени спадает пропорционально $\sqrt{P_{k}+P_{\mathrm{S}}}$ (рис. 9), где $P_{k}$ - заселенность возбужденного состояния выделенной моды. 


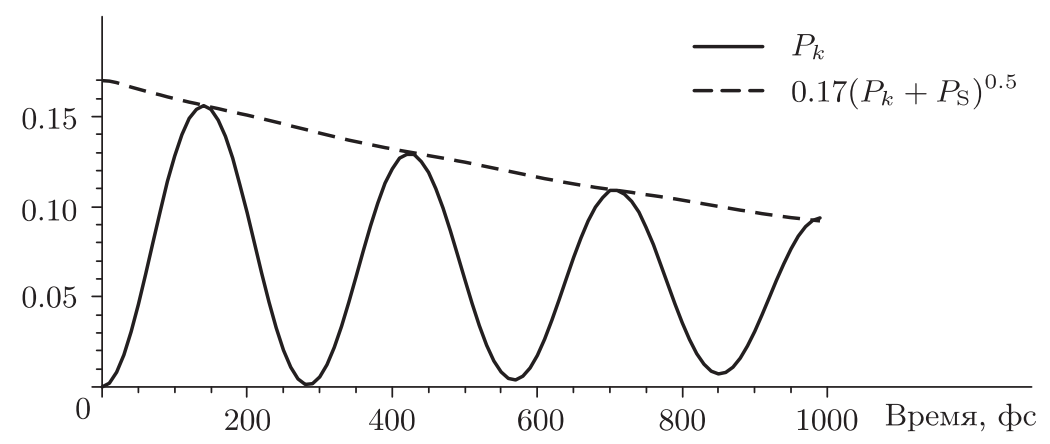

Рис. 9. Динамика заселенности возбужденного состояния выделенной моды термостата (сплошная линия). Штриховой линией показана временна́я зависимость корня суммы заселенностей возбужденных состояний наблюдаемой подсистемы и выделенной моды термостата.

Полученный результат показывает, что скорость термализации этой моды определяется не только ее состоянием, но также и состоянием наблюдаемой системы. Это, в свою очередь, подтверждает предположение о том, что термализация мод происходит за счет их взаимодействия с наблюдаемой подсистемой.

\section{6. ЗАКЛЮЧЕНИЕ}

В работе рассмотрена применимость основополагающего приближения диссипативной динамики, состоящего в неизменности состояния термостата для описания сверхбыстрых процессов в крупных биологических молекулах, в частности первичного переноса электрона в реакционном центре фотосинтеза пурпурных бактерий. На основе расчетов динамики модельных систем, приближенных к реальным, было показано, что использование этого приближения справедливо для описания как монотонной, так и осциллирующей динамики реакционной подсистемы в крупных биологических молекулах, а отказ от него не приводит к значительным изменениям и к каким-либо дополнительным эффектам в динамике.

Путем внесения в гамильтониан термостата малых дополнительных элементов, описывающих взаимодействие его мод между собой, было показано, что спектральная функция взаимодействия "система-термостат" может сильно изменяться, что в свою очередь может приводить к изменению динамики наблюдаемой подсистемы.

Показано также, что в системе с ограниченным числом степеней свободы термостата возможна его термализация за счет взаимодействия с наблюдаемой подсистемой, что позволяет отказаться от учета дальнего окружения наблюдаемой подсистемы при описании ее диссипативной динамики даже в случае, когда некоторые моды термостата значительно изменяют свое состояние.

Благодарности. Авторы благодарны профессору В. В. Еремину за ценные комментарии. Работа выполнена при финансовой поддержке РФФИ (гранты № 13-0300621 и 15-03-02604). 


\section{Список литературы}

[1] I. Kassal, A. Aspuru-Guzik, New J. Phys., 14:5 (2012), 053041, 13 pp.

[2] H. Lee, Y.-C. Cheng, G. R. Fleming, Science, 316:5830 (2007), 1462-1465.

[3] T. Ritz, S. Park, K. Schulten, J. Phys. Chem. B, 105:34 (2001), 8259-8267.

[4] A. G. Yakovlev, A. Y. Shkuropatov, V. A. Shuvalov, FEBS Lett., 466:2-3 (2000), 209-212.

[5] A. G. Yakovlev, V. A. Shuvalov, J. Theoret. Biology, 343 (2014), 92-101.

[6] D. Egorova, A. Kühl, W. Domcke, Chem. Phys., 268:1-3 (2001), 105-120.

[7] V. I. Novoderezkhin, A. G. Yakovlev, R. van Grondelle, V. A. Shuvalov, J. Phys. Chem. B, 108:22 (2004), 7445-7457.

[8] V. May, O. Kühn, Charge and Energy Transfer Dynamics in Molecular System, Wiley-VCH, Weinheim, 2004.

[9] U. Weiss, Quantum Dissipative Systems, World Sci., Singapore, 2008.

[10] A. G. Redfield, "The theory of relaxation processes", Advances in Magnetic Resonance, Advances in Magnetic and Optical Resonance, 1, ed. J.S. Waugh, Academic Press, New York, 1965, 1-32.

[11] К. Блум, Теория матрицы плотности и ее приложения, Мир, М., 1983.

[12] В. В. Поддубный, И. О. Глебов, В. В. Еремин, ТМФ, 178:2 (2014), 295-304.

[13] И. О. Глебов, В. В. Еремин, Биофизика, 57:4 (2012), 589-597.

[14] Z.-Q. Liu, X.-T. Liang, Chin. Phys. Lett., 28:8 (2011), 089201, 5 pp.

Поступила в редакцию 12.12.2014 